\title{
Metal-Like Gravity and Its Cosmological Applications
}

\author{
Kamal Barghout \\ Math and Science, Prince Mohammad Bin Fahd University, Alkhobar, Saudi Arabia \\ Email: kbarghout@pmu.edu.sa
}

Received 5 October 2014; revised 3 November 2014; accepted 27 November 2014

Copyright (C) 2014 by author and Scientific Research Publishing Inc.

This work is licensed under the Creative Commons Attribution International License (CC BY). http://creativecommons.org/licenses/by/4.0/

(c) (i) Open Access

\begin{abstract}
Modification to Newton gravitational interaction is presented. It provides an understanding of a novel universal gravitational field of particle origin that defines alternative attributes to elementary constituents of matter particles and the gravitational interactions between them. It investigates gravitational relationship between two types of mass. The model assigns Coulombic gravitational interaction to DM particles and baryons by attributing self-antigravity to both normal matter and dark matter (DM). It defines the interaction as like particles repel while unlike particles attract. Metal-like force is proposed where same type mass (baryons) are gravitationally attracted to each other when a sea of DM particles are attracted to them and glue them together analogous to a metal bond. At close range, other dominant forces take place such as electromagnetic force. In light of this model, intergalactic self-repulsive DM particles are proposed to result in accelerating expansion of the universe. The model produces flat rotational curves for spiral galaxies and provides a physical explanation to MOND theory.
\end{abstract}

\section{Keywords}

Dark Energy, Dark Matter, MOND, Non-Traditional Gravity

\section{Introduction}

It has always been assumed that anti-gravitational particles are nonexistent as their existence largely runs against observation. A recent paper investigated whether virtual gravitational dipoles could be a solution to the dark energy (DE) problem [1]. While the dipoles are described there by repulsive matter and antimatter particles, here repulsive gravitational force of same-type dark matter (DM) particles that permeate intergalactic space produces similar results. The analogy is similar as far as how gravitational dipoles of virtual repulsive matter-antimatter particles contribute to vacuum energy or DM gravitational self-repulsive nature as suggested in this paper. 
Most physicists reject the concept of antigravity and are inclined to think for example that matter and antimatter must have identical gravitational properties and will always attract [2]-[4]. Very few argue otherwise. Some argued that antigravity could in fact be a potential explanation for CP violation [5], where the author reconsidered the impossibility arguments against antigravity and tried to rehabilitate a form of antigravity where an apparent violation of energy conservation would be associated with entropy production.

Newtonian dynamics fail to explain gravitational potentials of galaxies and galaxy clusters by only considering their baryonic mass [6]. Proposed solutions either invoke dominant quantities of non-luminous dark DM [7] or modification to Newton's law [8]. For example, the problem of flattening of galaxy rotation curves inspired researchers to investigate modification of gravitational theories by introducing weakly or non-interacting matter fields [9]-[12]. While such theories successfully explain much of cosmological dynamics within the realm of GR, they failed to explain the source of such a field. In this paper, such a field is presented by modification of gravitational interaction of two types of mass-one is DM particles and the other is baryonic matter. Other works suggested that visible baryonic matter and hypothesized DM were spatially coincident in most of the universe [13] [14]. These works favored the DM hypothesis over modification of Newtonian gravity.

The nature of DM particles is a mystery. Particle physics models suggest that DM is either axions, which is characterized as hypothetical new particles associated with quantum chromodynamics, or WIMPs, hypothetical new particles with weak interactions or TeV-scale masses, neither of which have been detected.

If DM had self-anti-gravitational nature and DE shared the same origin, it would be an easy task to explain DE as simply the result of DM particles' gravitational self-repulsive nature in the intergalactic region, a perfect candidate to a cosmological constant. This assumption doesn't represent a challenge in galactic regions if DMbaryonic gravitational interaction is attractive as widely accepted. This is because heavy baryonic objects will attract relatively light DM particles and form halos. Furthermore, introducing a self-repulsive attribute of DM particles adds yet another scattering factor to DM particles trapped in a gravitational potential as in a galactic DM halo. In such a scenario, DM galactic halos provide the means for holding galaxies intact as observationally seen. This approach is discussed in this model.

On the other hand, presenting self-anti-gravitational attribute to DM particles necessitates similar anti-gravitational attribute to baryons opposite to observations. This new physics pushes towards a new look at the dynamics of baryons. The observational fact that the normal mass seems to have a self-attractive gravitational properties has always led our thinking to drop a Coulomb-like gravitational option. Here, positive and negative matters are redefined by this new gravitational interaction to produce positive and negative energy respectively. A composite baryon-DM matter produces negative gravitational potential energy while DM or baryonic mass standing alone produces positive gravitational potential energy. The dynamics of the universe is solely governed by the gravitational interaction of baryon-DM matter in the universe.

In this paper, a new modification to Newtonian gravity is compared to cosmological observation via compliance with flat rotation curves in spiral galaxies. It is hoped that by better understanding the gravitational interaction of dark matter a candidate for the cosmological dark matter particle can be identified. Also, dark energy and dark matter are unified. It is also proposed that gravity might have an electromagnetic origin since the theory proposes identical electric-like force mathematical form.

\section{Basis of the Model}

The model is stemmed from Newtonian-Coulombic approach where mass is similar to charge; like masses repel and unlike attract. It however presents a challenge to cosmological observation since baryons seem to show selfattractive behavior. It proposes a long range gravitational interaction with baryon-baryon repulsive gravitational interaction suppressed by a metal-like attraction mechanism. At close range, other forces, e.g., electromagnetic force, are responsible for bonding baryonic material. Consider two gravitationally interacting baryonic objects; the interaction will be repulsive as suggested. Only due to the existence of attractive force between a sea of opposite-mass particles (DM particles) and baryonic objects, the baryonic-baryonic interaction will be seen as attractive. This approach seems plausible if it can explain the dynamics of general relativity (GR) in the weak field of Newtonian limits [15].

\section{Composite DM-Baryonic Matter}

DM-particles are proposed to form a DM-halo around baryonic objects. Such a DM-baryon composite necessar- 
ily requires that DM doesn't annihilate with the baryonic core. It is assumed that DM-baryonic interaction and DM-self interaction is gravitational. It is further assumed that a complete DM-halo drag occurs. A composite DM-baryonic object forms when baryonic entity gravitationally attract relatively small DM-particles and forms gravitationally neutral and stable object. A metal-like bond is a bond that forms between two composite DMbaryonic objects when their DM-halos overlap thereby forming yet larger composite objects. Any gravitationally bound structure, e.g. cosmic structures such as galaxies, is suggested to be made when their sub-composite DMbaryonic objects are gravitationally bonded by the proposed metal-like bond. Composite objects are just referred to as baryonic objects in the remaining text.

\section{Feasibility of Metal-Like Force}

The proposed metal-like force should be directional along the line connecting the centers of the two baryonic objects. This could be seen for an isolated two baryonic objects as the cloud of particles of opposite type would have highest probability density between the two objects as Figure 1 illustrates.

A simple analogy is a person acting as the overlapping DM mass, pulling two friends by both hands. Increasing his pull to his friends increases the apparent attractive force between the two friends. The net centered DMmass between the two baryonic objects can be viewed as an overlapping of the DM halos of the two baryonic objects as Figure 2 shows.

The close relationship between the DM halo particles and the baryonic galactic content can be readily explained by the intimate DM-baryonic attractive relationship as well as the DM self-repulsive nature as presented here. For cosmological large structures such as galaxies, Figure 3 illustrates baryonic core with a baryonic edge star of a hypothetical spherically symmetrical galaxy. The DM halo particles are included in the volume between the edge star and the baryonic core.

Three gravitationally bound systems can be clearly distinguished employing the DM-baryonic gravitational relationship. First, Newtonian behavior exists within the baryonic galactic inner cores with a constant metal-like force due to constant DM-density on average. Second, a metal-like varying gravitational force exists away from galactic cores due to varying DM halo density. Third, a centered DM halo exists in a cluster of galaxies as
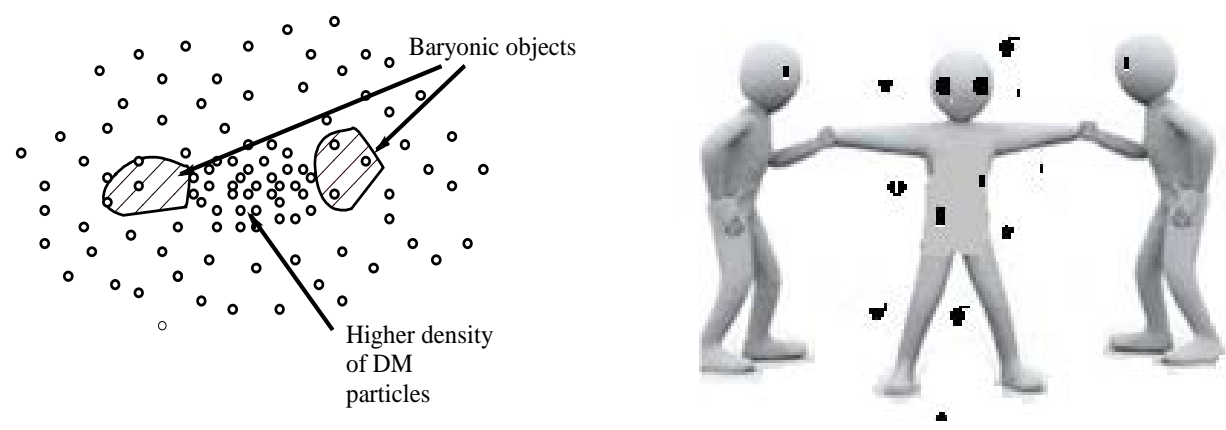

Figure 1. Higher density of DM particles between two baryonic objects.

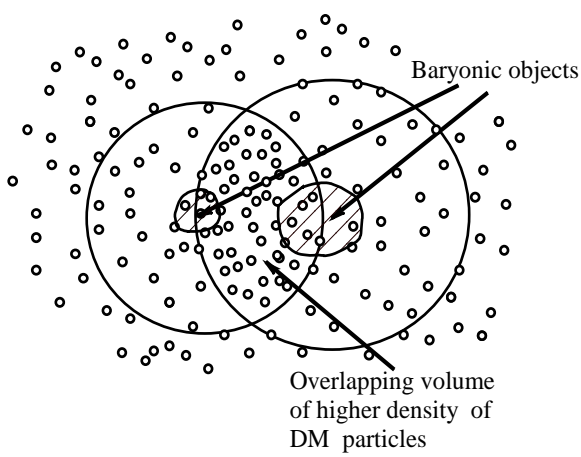

Figure 2. Higher density of DM particles in the overlapping volume of two DM halos. 


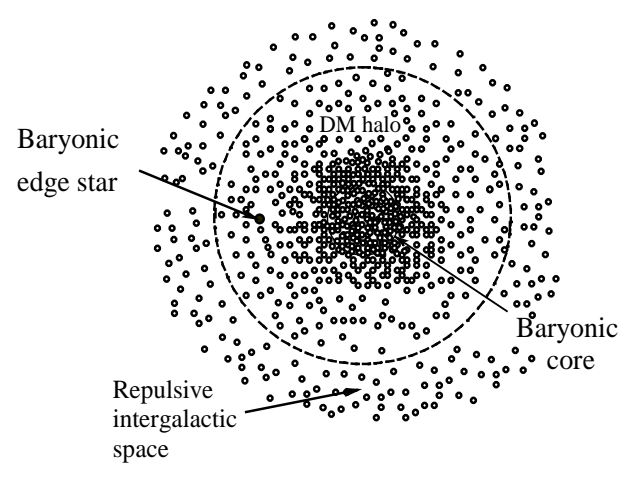

Figure 3. Galactic system showing intergalactic space, an edge-star, the core and the enclosed DM halo.

illustrated in Figure 4. Here, the overlapping volume due to all of the DM-halos of the cluster's sub-galaxies constitutes the attracting agent to the galaxies which represents the cluster's "dark core".

\section{Metal-Like Force Mathematical Form}

Metal-like force approach is an attempt to explain the dynamics of the universe via modification to Newtonian gravity. In a metallic bond, the force that describes ionic interaction can be described as Coulombic and proportional to $r^{-2}$. Likewise, the gravitational force that describes the proposed metal-like force can be described as proportional to $r^{-2}$ as we see it in Newtonian form. Therefore the magnitude of the gravitational force can be described by the equation,

$$
F_{g}=G \frac{\left|m_{1} m_{2}\right|}{r^{2}}
$$

where $G$ is the gravitational constant, $F_{g}$ is the gravitational force, $m_{1}$ and $m_{2}$ are the baryonic/DM masses.

\section{Stability of Cosmic Structures}

In the metal-like model, any stable cosmic structure should be gravitationally bound with DM particles as the acting bonding agent. In the inner galactic core, the metal-like force results in Newtonian dynamics while in the galactic outskirts the metal-like force predicts different dynamics. For nucleation of any cosmological structure, DM halos of baryonic objects should overlap to initiate the metal-like bonding process which produces a permanent gravitationally bound structure.

\section{Metal-Like Cosmology}

Metal-like gravity (MLG) produces attractive force between the overlapping DM mass and the baryonic cores of two interacting baryonic objects, e.g. between a galaxy and an edge-star. The overlapping DM mass has two components; one by the galaxy which is responsible for attracting the star to the overlapping volume and one by the star which attracts the galactic core to the overlapping volume. The former is responsible for bonding the star to the galaxy. It is proposed here that a "baryonic" edge star's dynamics is mainly determined by the proposed metal-like gravitational relationship. Just as in the galactic inner core, the metal-like interaction is responsible for bonding the edge-star to the galactic core with the exception that the DM bonding particles are not distributed evenly throughout the galactic halo as in the core. Instead, the halo-DM density decreases proportional to the distance from the core due to the self-repulsive nature of the DM particles as illustrated in Figure 5.

Here, the DM-halo is treated classically with DM particles constitute a DM-hydrostatic fluid of which gravitational collapse of the halo is counterbalanced by hydrostatic pressure made by the self-repulsive gravitational nature of the DM-particles. At particle level, the DM-baryonic interaction is assumed to prevent annihilation or singularity collapse, hence supporting a build-up of pressure. Of special importance is that bulk DM-particles' spacing, which defines the halo's local density, is totally governed by the induced DM-hydrostatic pressure and 


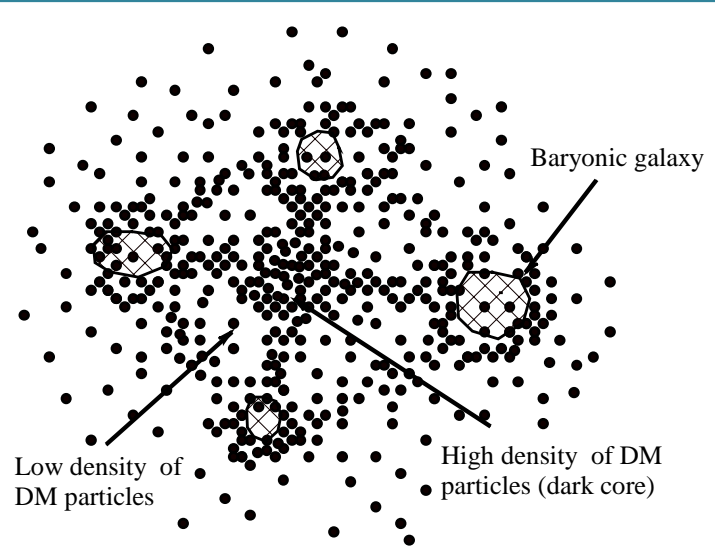

Figure 4. DM particle distribution in a cluster of galaxies.

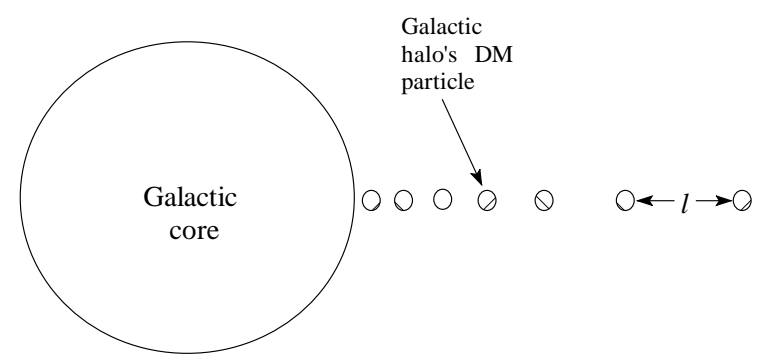

Figure 5. The galactic DM halo density is proportional to the halo's DM particles' spacing $(l)$ at the edge-star's location (not to scale).

inversely proportional to the altitude from the surface of the galaxy. An analogy is the atmospheric pressure "electrically" induced to counterbalance a gravitational collapse of atmospheric air molecules under the acceleration of gravity leading to compression of air molecules with air denser near the Earth's surface. Galactic DM halo gravitational condensation leads to its density inversely proportional to the spacing $(l)$ between two neighboring DM halo particles in the radial direction from the galactic center as illustrated in Figure 5 above. Considering a universal DM particle's mass, the halo-DM particle's dynamics can be approximated by a gravitationally balanced repulsive Newtonian force between the DM particle and the one preceding it from the galactic core with the attractive force between the DM particle and the galactic core. The gravitationally induced "DMhydrostatic" pressure gradient force balances with the core-DM attractive force to produce the equation $m / l^{2}=M_{l} / r^{2}$; where $r$ is the distance to the center of the galaxy, $m$ is the mass of the DM particle and $M_{l}$ is the galactic luminous mass. Here, the repulsive force between the DM-particle and the one preceding it is taken as first order approximation. The equality produces the ratio $\sqrt{m} / l$ that describes the halo's "DM-linear" mass density (square root of DM particle's mass per spacing $l$ ) in the galactic radial direction as,

$$
\sqrt{m} / l=\sqrt{M_{l}} / r .
$$

To approximate the galactic halo's DM-density within the overlapping volume, the DM particle's spacing $l$ changes according to the location of the overlapping volume relative to the galactic core $(r)$. Taking $l$ as an arbitrary unit vector of the chosen coordinate system at an arbitrary location of the edge-star, the galactic DM density within the overlapping volume $\rho_{D}=(\sqrt{m} / l) \cdot\left(\lambda / l^{2}\right)$, where $\lambda$ is a constant. The term $\left(\lambda / l^{2}\right)$ describes a constant DM surface density (square root of DM particle's mass per l-spacing squared) as recently discovered [16]. This is simply because the only variation in galactic halo's DM-density is in the radial direction due to the varying core-DM attractive force. This leads to the density to be invariable in other directions hence the term with a constant value. Employing Equation (2), the galactic halo's DM-density can be approximated by $\rho_{D}=\delta \sqrt{M_{l}} / r$, where $\delta$ is a constant equals $\lambda / l^{2}$. 


\section{Galactic Pull on an Edge-Star}

To derive the gravitational force between an edge-star and a galactic core, the galaxy and the edge-star are represented as two interacting baryonic objects with their DM halos overlapping. As Figure 6 shows, the overlapping DM particles pull on the star as well as on the galaxy. Its pull on the star is what is seen as the pull by the galaxy on the star and that is what determines the dynamics of the stars as seen in their rotational velocities. The repulsive force between the galactic baryonic core and the star's baryonic core is always smaller than the net attractive force between the two baryonic cores. To see this for a star-star system, for each star the extra DM mass in the overlapping volume from the other star projects the same magnitude of DM mass in the core of the star to preserve mass neutrality since the DM halo shields baryonic mass in the core. Therefore, summing over the two attractive forces between the stars' net baryonic cores and the DM mass in the overlapping volume always yields a larger force than the repulsive force between the two "net" baryonic cores. Key factors that define the galactic pull on a star are the star's DM component in the overlapping volume $\left(S_{D}\right)$ and the galactic DM component in the overlapping volume $\left(G_{D}\right)$. As Figure 6 illustrates, $S_{D}$ pulls on the galactic baryonic core with negligible small magnitude due to the large distance to the core as well as the small galactic baryonic mass of the galaxy due to the shielding of the core by galactic DM halo, and $G_{D}$ pulls on the star with a significant magnitude. It is the pull by $G_{D}$ on the star that is seen as the pull by the galaxy on the star. The pull by $S_{D}$ on the galactic baryonic core only becomes significant when the system is a star-star one rather than a star-galaxy system.

Considering a universal one-type of DM particle, the DM particles that constitute both $S_{D}$ and $G_{D}$ are indistinguishable. Furthermore, for an edge star the DM overlapping volume is invariable since the non-overlapping volume will always remain very small and negligible since the edge-star is far away from the galactic core. Therefore the overlapping volume is defined solely by the star's DM halo and remains invariable relative to the location of the star from the galactic core, as Figure 7 illustrates.

The DM density within the invariable overlapping volume is dominated by the edge-star's strong gravitational potential which results in an invariable $S_{D}$ density but a variable $G_{D}$ density (depending on the distance of the edge-star to the galactic core) governed by Equation (2) and illustrated by Figure 8.
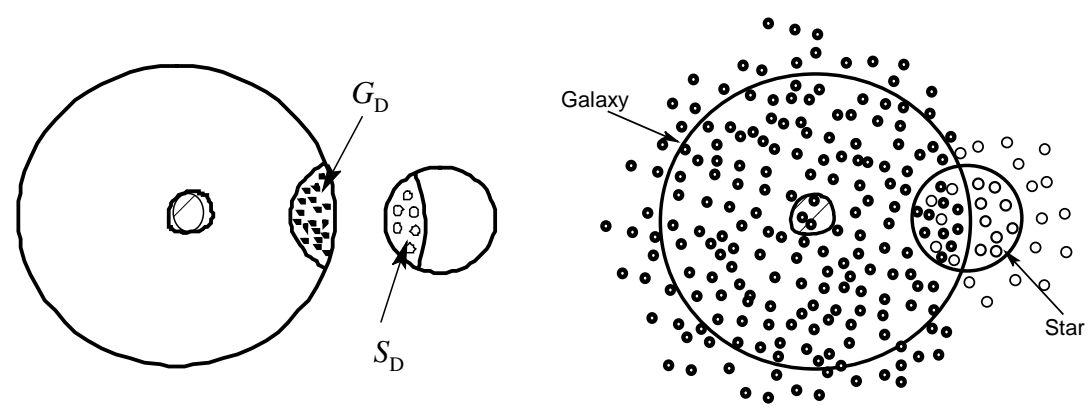

Figure 6. The attractive force a galactic core applies on an edge-star comes from the attractive force between the star's baryonic core and $G_{D}$ (not to scale).
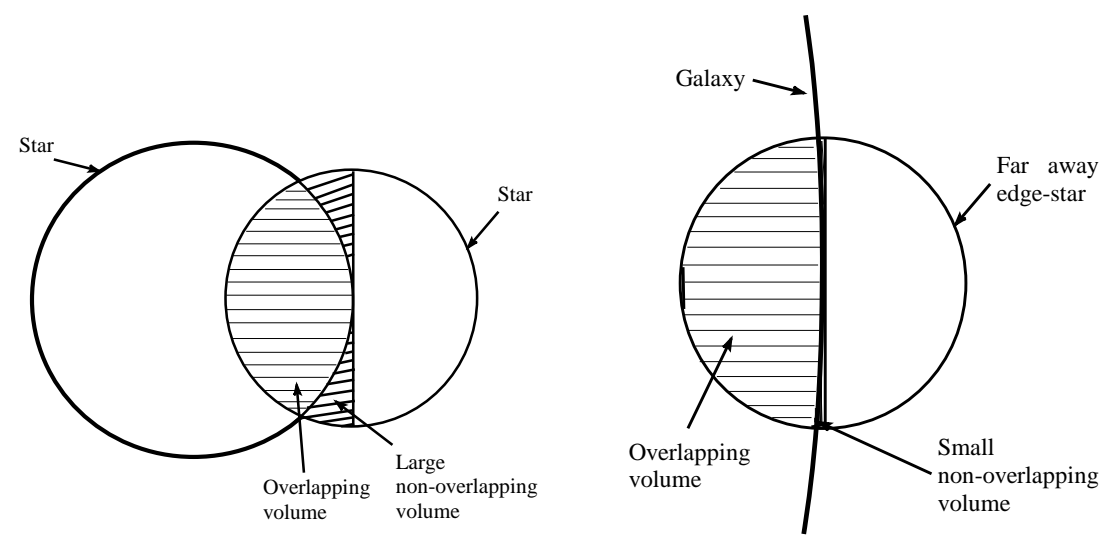

Figure 7. Overlapping volume of DM halos is invariable for an edge star in a galaxy (right), and smaller for Newtonian galactic core sub-structure of two stars (left). 


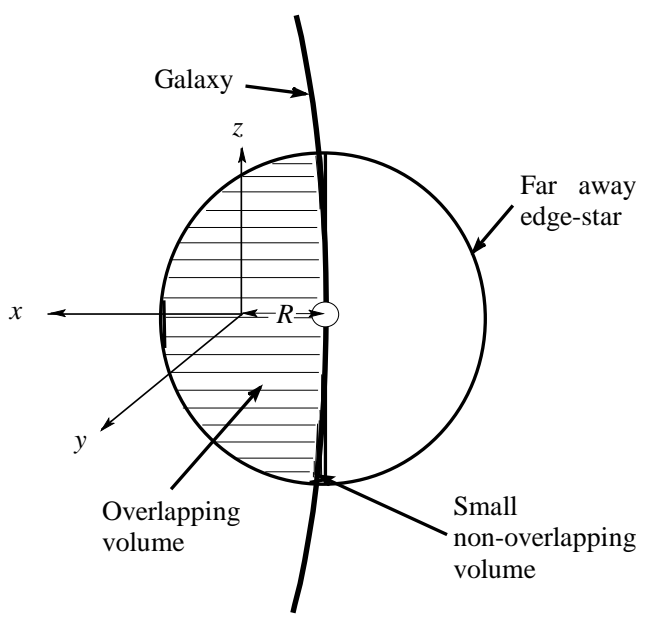

Figure 8. $G_{D}$ density changes in the $x$-direction; the direction to the galactic center.

The pull by the galactic DM in the overlapping volume, $G_{D}$, on the edge-star is then determined by,

$$
f_{g}=\frac{G M_{S} G_{D}}{R^{2}}
$$

where $M_{S}$ is the edge-star's baryonic mass and $R$ is the "invariable" distance between the star and the center of the overlapping volume (see Figure 8 above). The only variable in Equation (3) above is $G_{D}$, which is determined by the product of its density $\rho_{D}$ and the "invariable" overlapping volume. To describe the rotational velocity of the edge-star, we need to map the centripetal force of the edge-star around the center of mass of the overlapping volume to that around the center of the galaxy. The configuration of the MLG star-galaxy system forces us to map the centripetal force to relate it to the galactic center as the DM particles of the $G_{D}$ mass that accounts for the centripetal force are always aligned along galactic radial direction, with a radial line of force maintained by halo DM particles.

As an illustrating example, picture a star entering a galactic DM halo from outside due to its inertia. The galactic $G_{D}$ attracts the newly entering star's baryonic mass, thereby dragging the star inward. Figure 9 shows the overlapping volume rotating along with the star around the galactic core, continually pulling the star radially inward, maintaining the MLG bond.

Turbulent DM-fluid collision process seems to occur in the overlapping volume due to self-repulsive colliding DM particles accompanied by the high relative speed of the star.

Mapping the edge-star's centripetal force to the galactic center, Equation (3) becomes,

$$
\frac{v^{2}}{r}=\frac{\beta G \sqrt{M_{1}}}{R^{2} r}
$$

where $G_{D}$ is replaced by $\beta \sqrt{M_{l}} / r$, representing the galactic DM-density in the overlapping volume; $\rho_{D}$ multiplied by the non-varying overlapping volume as obtained from Equation (2) above, where $\beta$ is a constant equals $\delta$ multiplied by the invariable overlapping volume.

Equation (4) can be reduced to,

$$
v=\sqrt{\alpha G\left(M_{l}\right)^{0.5}}
$$

where $\alpha$ is a constant equals $\beta / R^{2}$.

\section{MLG, MOND and Rotation Curves}

The rotation curves (RCs) of spiral galaxies become approximately flat at the largest radii observed [17] [18]. This is one of the strongest indications of the need for dynamically dominant DM in the universe. The inner shape of the rotation curves is well predicted by the distribution of observed baryons [19]. There appears to be 


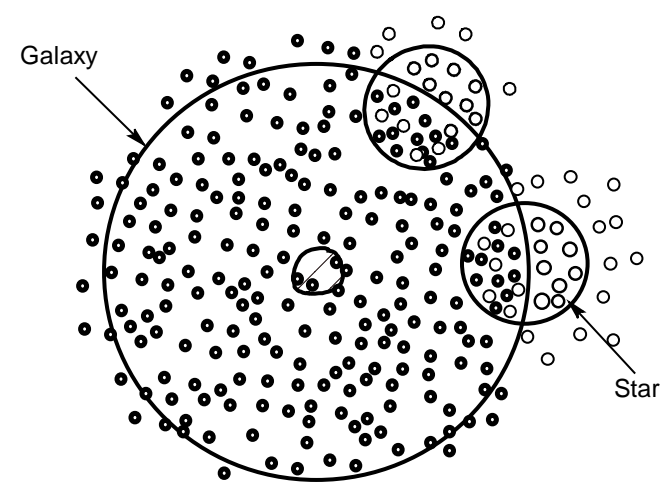

Figure 9. Overlapping volume rotates with the star.

though a characteristic acceleration scale at which baryonic material alone can no longer account for the observed dynamics, hence the need for a DM halo in galaxies [20]. The universality of the RCs in combination with the invariant distribution of the luminous matter implies a universal DM distribution with luminositydependent scaling properties [21], i.e., luminosity, dictates the rotational velocity at any radius for any object, so revealing the existence of a universal RC. Modified Newtonian dynamics (MOND) [8] is a theory that describes the rotational velocity of stars far away from the galactic center without the presence of DM. It proposes a modification of Newtonian gravity to explain flat galactic rotation curves (RCs) by introducing the function $\mu\left(a / a_{0}\right)$ which equals one at normal acceleration and equals $\left(a / a_{0}\right)$ at very low acceleration; where $a$ is the centripetal acceleration of the star and $\alpha_{0}$ is a constant of about $1.2 \times 10^{-10} \mathrm{~m} / \mathrm{s}^{2}$ characteristic of MOND's low acceleration. MOND expresses the galactic rotational velocity of stars as $\sqrt[4]{G M_{l} a_{0}}$. MOND's empirical formula is very successful in fitting observational data of galactic flat RCs. A possible physical interpretation of this functional form is provided by the assumption that gravity is mediated by gravitons with non-zero mass. The suggested DM-particle with gravitational characteristic as described here is a good candidate for the graviton. Metal-like cosmology provides a physical explanation to MOND theory. The configurational analysis of Newtonian approach of MLG theory is crucial to determine galactic dynamics as described by Equation (5). Such a power law of Equation (5) that has only one free fitting parameter explains the intimate relationship between luminous mass of galaxies and DM halo densities obtained from observations. Current CDM halo models express DM densities as power laws. For example, theoretical DM halos produced in computer simulations are best described by the Einasto profile $\rho(r)=\rho_{0} \mathrm{e}^{-\alpha r^{n}} \quad$ [22]. While those models don't seem to explicitly offer a physical interpretation of this fine tuning of DM-luminous mass dependency as satisfied by the observed TullyFisher-type relations, this is readily explained in the metal-like model.

To compute the rotational velocity of an edge-star, we employ Equation (5) and choose the magnitude of the fitting parameter $\alpha$ as 1.345 for spiral galaxies. Equation (5) expresses the rotational velocity curves of galaxies as flat and produces identical RC curves as MOND for galaxies. It is worthwhile to note that unlike MOND which is a DM-free theory, metal-like cosmology differentiates between the DM-distribution in a galaxy and DM-distribution in a cluster of galaxies as shown in Figure 4. Equation (5) does not apply to clusters of galaxies which explain the failure of MOND's theory to explain the dynamics of clusters of galaxies. Only if the system of the cluster of galaxies consists of a core extra-large galaxy with varying DM halo, Equation (5) may apply. To compare with MOND, the luminous mass was derived from a perfect fit to the rotation curves of galaxy NGC 224 [23] using MOND formula (assuming MOND produced a perfect fit). Rotation curves were then generated by MLG using Equation (5), which produced identical rotation curves as MOND (see Figure 10).

Since MOND is widely rejected as a non-DM based theory but yet it is very successful in fitting RCs for spiral galaxies, one can ask, shouldn't there be DM-based model that explains the success of MOND? Metal-like gravity is just that model. MOND and MLG produce the same galactic RCs, therefore the fitting parameter $\alpha$ can be derived from MOND formula which is found to be $\sqrt{a_{0} / G}$.

Equation (5) above actually does not describe flat rotation curves. Instead, RCs should rise "slowly". This can be seen from MLG if the inflation of the overlapping volume upon moving of an edge-star toward the galactic center is taken into consideration. The inflation occurs because the "self-repulsive" DM particles of the star trapped in the overlapping volume $\left(S_{D}\right)$ find higher gravitational potential closer to the galactic center and 


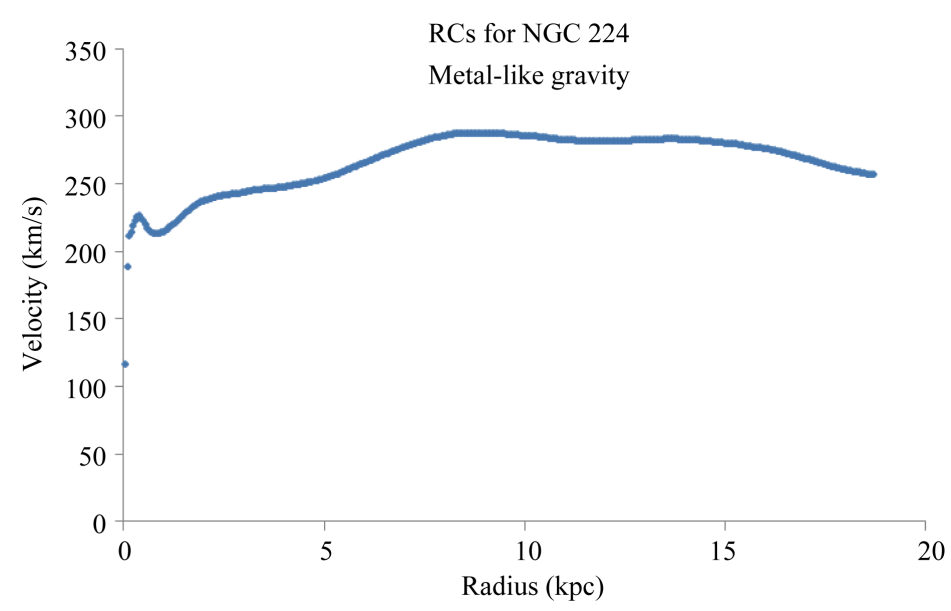

Figure 10. MLG produces identical RC curves for galaxy NGC 224 as MOND.

therefore higher density of the galactic DM particles in the overlapping volume $\left(G_{D}\right)$. $G_{D}$ particles inflate the overlapping volume by dispersing the $S_{D}$ particles. This results in shifting the center of DM-mass of the overlapping volume towards the galactic center and increasing the distance to the center of the star, effectively reducing the gravitational force $\left(F_{g}\right)$, thereby reducing the rotational velocity. The increase in $R$ is progressive and small considering the large relative distance to the galactic center. This results in slowly rising RCs.

\section{Dark Energy}

Metal-like gravity can safely explain the most perplexing mysteries, the nature of DM and DE, since it can explain the way normal matter in galaxies and clusters of galaxies behave. Under MLG model, the source of the DE field that results in the accelerated expansion of the universe is an excess of DM particles existing in the intergalactic regions. The self-repelling nature of the DM particles leads to this acceleration. Since the model necessitates equal amounts of normal matter and dark matter to explain the galactic dynamics, DM particles that exist in the intergalactic regions must be excess particles over normal matter. A potential method that may have produced excess of DM particles over normal particles at the time of the Big Bang event is a CP violation process. At this point it is compelling to think of a DM particle candidate as an antimatter one. The only reason that drives us away from that direction is the absence of the annihilation signature of the DM particle with normal matter.

Furthermore, studying the cosmological constant under the metal-like cosmology during the evolution of the universe sheds light on the magnitude of the DM particle's mass. This can be done by relating the cosmological constant to a hydrostatic pressure of a DM-fluid in the intergalactic region and defining it as the quantum vacuum in that region. Metal-like cosmology provides the means to explain the huge difference of a factor of about $10^{-120}$ between the measured cosmological constant and that described by an effective local quantum field theory.

\section{Conclusion}

A gravity model that describes dark matter (DM) and normal matter as two opposite types of mass that follow Coulomb law, where like particles gravitationally repel and unlike particles attract, is investigated. DM particles are proposed to permeate all of space in the observable universe. The model describes DM particles as selfrepelling, resulting in accelerated expansion of the universe due to the DM presence in the intergalactic regions. The model describes a "metal-like" force that suppresses baryonic self-repulsive gravitational nature and introduces Newtonian regime within galactic cores. It describes DM-based galactic dynamics that explains MOND's success in describing flat RC curves. The model introduces new physics and can explain many cosmological mysteries such as the nature of dark matter and dark energy. The model's apparent contradiction to well established doctrines of physics just as GR should be seriously addressed. 


\section{Acknowledgements}

The author is grateful to the research-support from Prince Mohammad University.

\section{References}

[1] Hajdukovic, D.S. (2012) Quantum Vacuum and Virtual Gravitational Dipoles: The Solution to the Dark Energy Problem? arxiv/1201.4594

[2] Morrison, P. (1958) American Journal of Physics, 26, 358-368. http://dx.doi.org/10.1119/1.1996159

[3] Schiff, L.I. (1958) Physical Review Letters, 1, 254-255. http://dx.doi.org/10.1103/PhysRevLett.1.254

[4] Schiff, L.I. (1959) Proceedings of the National Academy of Sciences, 45, 69. http://dx.doi.org/10.1073/pnas.45.1.69

[5] Chardin, G. and Rax, J.M. (1992) Physics Letters B, 282, 256-262. http://dx.doi.org/10.1016/0370-2693(92)90510-B

[6] Zwicky, F. (1937) Astrophysical Journal, 86, 217. http://dx.doi.org/10.1086/143864

[7] Oort, J. (1932) Bulletin of the Astronomical Institutes of the Netherlands, 6, 249.

[8] Milgrom, M. (1983) Astrophysical Journal, 270, 365-370. http://dx.doi.org/10.1086/161130

[9] Trimble, V. (1987) Annual Review of Astronomy and Astrophysics. 25, 425-472. http://dx.doi.org/10.1146/annurev.aa.25.090187.002233

[10] Bergstrom, L. (2000) Reports on Progress in Physics, 63, 793-841. http://dx.doi.org/10.1088/0034-4885/63/5/2r3

[11] Hoekstra, H., Yee, H.K.C. and Gladders, M.D. (2004) The Astrophysical Journal, 606, 67-77. http://dx.doi.org/10.1086/382726

[12] Bertone, G. and Hooper, S.J. (2005) Physics Reports, 405, 279-390. http://dx.doi.org/10.1016/j.physrep.2004.08.031

[13] Buote, D.A., Jeltema, T.E., Canizares, C.R. and Garmire, G.P. (2002) The Astrophysical Journal, 577, $183-196$. http://dx.doi.org/10.1086/342158

[14] Gavazzi, R. (2002) New Astronomy Review, 46, 783-789. http://dx.doi.org/10.1016/S1387-6473(02)00246-4

[15] Gentile, G., Famaey, B., Zhao, H.S. and Salucci, P. (2009) Nature, 461, 627-628. http://dx.doi.org/10.1038/nature08437

[16] Rubin, V.C., Thonnard, N. and Ford, W.K. (1980) The Astrophysical Journal, 238, 471. http://dx.doi.org/10.1086/158003

[17] Bosma, A. (1981) The Astrophysical Journal, 86, 1791-1724. http://dx.doi.org/10.1086/113062

[18] Palunasand, P. and Williams, T.B. (2000) The Astrophysical Journal, 120, 2884-2903. http://dx.doi.org/10.1086/316878

[19] McGaugh, S.S. (2004) The Astrophysical Journal, 609, 652-666. http://dx.doi.org/10.1086/421338

[20] Salucci, P. and Burkert, A. (2000) The Astrophysical Journal, 537, L9-L12. http://dx.doi.org/10.1086/312747

[21] Graham, A.W., Merritt, D. and Diemand, J. (2006) The Astronomical Journal, 132, 2711-2716.

[22] Sofue, Y., Tutui, Y., Honma, M., Tomita, A., Takamiya, T., Koda, J. and Takeda, Y. (1999) The Astrophysical Journal, 523, 136-146. http://dx.doi.org/10.1086/307731

[23] Matthews, L.D., Gallagher III, J.S. and Van Driel, W. (1999) Astronomical Journal, 118, 2751-2766. http://dx.doi.org/10.1086/301128 
Scientific Research Publishing (SCIRP) is one of the largest Open Access journal publishers. It is currently publishing more than 200 open access, online, peer-reviewed journals covering a wide range of academic disciplines. SCIRP serves the worldwide academic communities and contributes to the progress and application of science with its publication.

Other selected journals from SCIRP are listed as below. Submit your manuscript to us via either submit@scirp.org or Online Submission Portal.
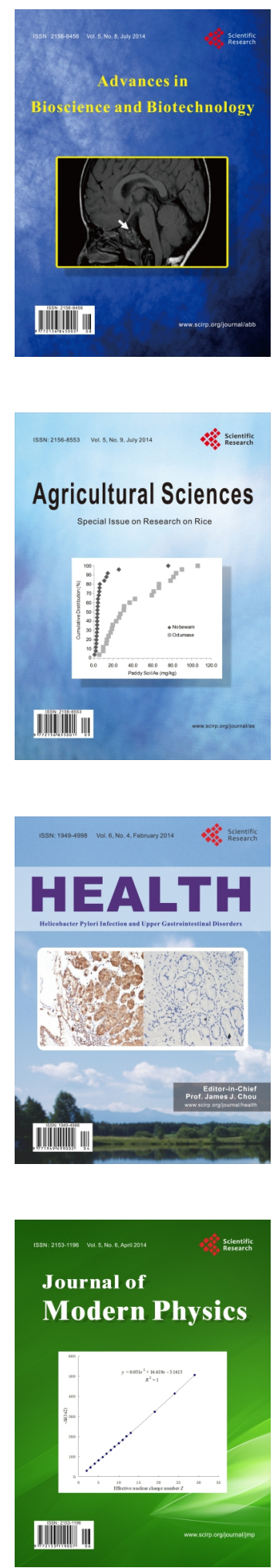
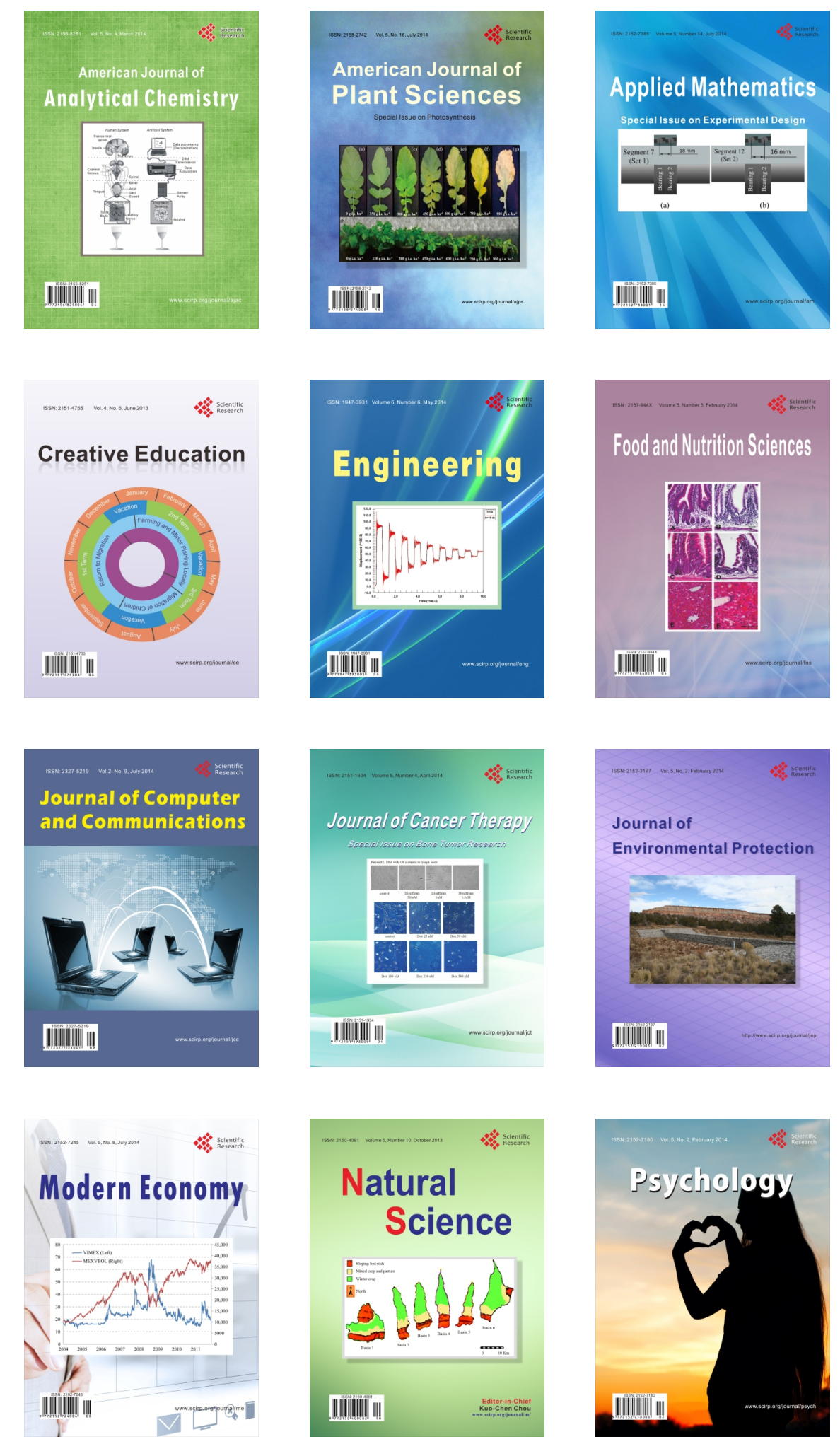